\title{
Results of using permanent magnets to suppress Josephson noise in the KAPPa SIS receiver
}

Caleb H. Wheeler, Marko Neric, Christopher E. Groppi, Matthew Underhill, Hamdi Mani, et al.

Caleb H. Wheeler, Marko Neric, Christopher E. Groppi, Matthew Underhill, Hamdi Mani, Sander Weinreb, Damon S. Russell, Jacob W. Kooi, Arthur W. Lichtenberger, Christopher K. Walker, Craig Kulesa, "Results of using permanent magnets to suppress Josephson noise in the KAPPa SIS receiver," Proc. SPIE 9914, Millimeter, Submillimeter, and Far-Infrared Detectors and Instrumentation for Astronomy VIII, 99141W (20 July 2016); doi: $10.1117 / 12.2231358$

Event: SPIE Astronomical Telescopes + Instrumentation, 2016, Edinburgh, United Kingdom 


\title{
Results of using permanent magnets to suppress Josephson noise in the KAPPa SIS receiver
}

Caleb H. Wheeler ${ }^{\mathrm{a}}$, Marko Neric ${ }^{\mathrm{a}}$, Christopher E. Groppi ${ }^{\mathrm{a}}$, Matthew Underhill ${ }^{\mathrm{a}}$, Hamdi Mani ${ }^{\mathrm{a}}$, Sander Weinreb ${ }^{\mathrm{b}}$, Damon S. Russell ${ }^{\mathrm{c}}$, Jacob W. Kooi ${ }^{\mathrm{b}}$, Arthur W. Lichtenberger ${ }^{\mathrm{d}}$, Christopher K Walker ${ }^{\mathrm{e}}$, Craig Kulesa ${ }^{\mathrm{e}}$

${ }^{a}$ School of Earth and Space Exploration, Arizona State University, 781 E Terrace Rd, Tempe, AZ,

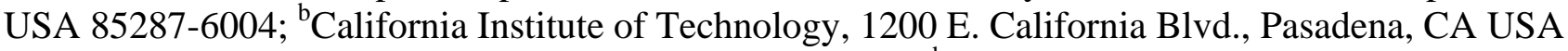
91125; ' Jet Propulsion Laboratory, Pasadena, CA, USA; ${ }^{\mathrm{d} U n i v e r s i t y ~ o f ~ V i r g i n i a, ~ D e p a r t m e n t ~ o f ~}$ Electrical and Computer Engineering, 351 McCormick Rd., Charlottesville, VA, USA 22904-4743; ${ }^{\mathrm{e}}$ University of Arizona, Steward Observatory, 933 N. Cherry Ave., Tucson, AZ, USA 85721

\begin{abstract}
We present the results from the magnetic field generation within the Kilopixel Array Pathfinder Project (KAPPa) instrument. The KAPPa instrument is a terahertz heterodyne receiver using a Superconducting-InsulatingSuperconducting (SIS) mixers. To improve performance, SIS mixers require a magnetic field to suppress Josephson noise. The KAPPa test receiver can house a tunable electromagnet used to optimize the applied magnetic field. The receiver is also capable of accommodating a permanent magnet that applies a fixed field. Our permanent magnet design uses off-the-shelf neodymium permanent magnets and then reshapes the magnetic field using machined steel concentrators. These concentrators allow the use of an unmachined permanent magnet in the back of the detector block while two small posts provide the required magnetic field across the SIS junction in the detector cavity. The KAPPa test receiver is uniquely suited to compare the permanent magnet and electromagnet receiver performance. The current work includes our design of a ' $U$ ' shaped permanent magnet, the testing and calibration procedure for the permanent magnet, and the overall results of the performance comparison between the electromagnet and the permanent magnet counterpart.
\end{abstract}

Keywords: SIS junction, Heterodyne, KAPPa, Array Receivers, THz

\section{INTRODUCTION}

Using a technique known as mixing, Superconducting-Insulating-Superconducting (SIS) devices in terahertz (THz) heterodyne receivers allow for the down-conversion of high frequency signals (RF) to lower, intermediate frequencies (IF). The IF can then be transmitted and analyzed by commercially available components. For an SIS device to have efficient down-conversion at high frequencies, an applied magnetic field is required to suppress Josephson Noise [1], or Cooper pairs tunneling across the SIS junction. Down conversion is most efficient when the applied magnetic field creates an integer number of magnetic flux quanta in the superconducting regions of the SIS junction. However, there is a considerable degradation of performance for non-integer flux quanta applied magnetic fields. Further, the KAPPa receiver has very poor response when no applied field is present. The historic choice for magnetic field generation within an SIS receiver has been electromagnets because they allow for precise tuning of the magnetic field. However, electromagnets are too large and consume too much power to be used in future high pixel-count SIS array receivers. Our strategy to solve the issue of power consumption and packing density for future $\mathrm{THz}$ arrays is to replace a tunable electromagnet with an off-the-shelf fixed permanent magnet systems for magnetic field generation.

KAPPa makes the technological advancements needed to create arrays of $\mathrm{THz}$ heterodyne SIS receivers that are on the order of $\sim 1000$ pixels. For comparison, SuperCam is currently a state-of-the-art SIS receiver with a 64-pixel array [2]. Many technological improvements have been made by the KAPPa team to realize the future of large heterodyne arrays [3], [4]. To make an array with larger numbers of pixels, we are required to make each pixel smaller, simpler, and cheaper. The fulcrum of this work has been whether the magnetic field generation required for SIS receivers can be simplified without substantial degradation of performance that would negate the benefits of large array construction.

The KAPPa receiver was designed to house either an electromagnet or a permanent magnet to compare the performance with different modes of magnetic field generation. The flexibility of the KAPPa receiver allows for an electromagnet and

Millimeter, Submillimeter, and Far-Infrared Detectors and Instrumentation for Astronomy VIII,

edited by Wayne S. Holland, Jonas Zmuidzinas, Proc. of SPIE Vol. 9914, 99141W

(c) 2016 SPIE · CCC code: $0277-786 \mathrm{X} / 16 / \$ 18 \cdot$ doi: $10.1117 / 12.2231358$ 
permanent magnet system to be installed interchangeably from the backside of receiver's detector block. Installation from the backside negates the need to open the detector block and disturb the SIS device or supporting electronics. This feature provides a direct comparison of the same system's performance and an unambiguous test-bed for different magnetic field generation techniques. While the permanent magnets apply a fixed magnetic field, there are many ways to adjust the field strength of a magnet prior to installation in the receiver.

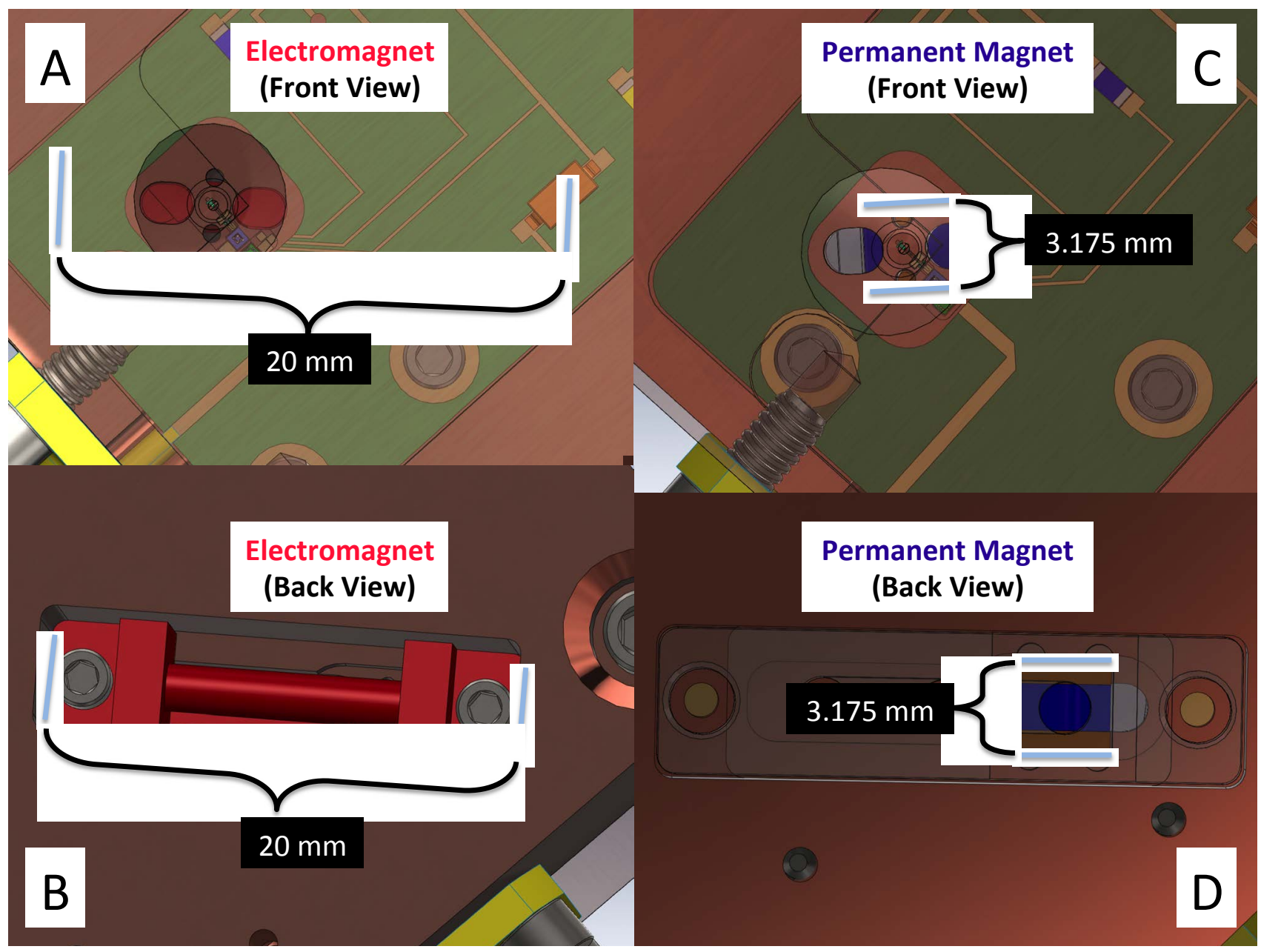

Figure 1. Solid models of the electromagnet and permanent magnet systems. The electromagnet footprint, $20 \mathrm{~mm} \times 5 \mathrm{~mm}$, viewed from the front (panel A) and the back (panel B) and the permanent magnet footprint $3.175 \mathrm{~mm} \times 3.175 \mathrm{~mm}$, viewed from the front (panel C) and the pack (panel D) of the detector block. The detector block houses the SIS junction and supporting electronics. In the front view, the feedhorn block has been made translucent, however the outline of $6 \mathrm{~mm}$ diameter feedhorn aperture can still be seen. In panel $\mathrm{D}$, The permanent magnet holder has been made translucent. The permanent magnet holder is needed to reuse the same mounting scheme required by the electromagnet.

\section{MAGNET DESIGN}

\subsection{Benchmark Electromagnet}

The electromagnet used as a benchmark in the KAPPa project was originally designed for SuperCam 64-pixel heterodyne focal-plane array. The SuperCam team, through considerable effort, minimized the manufacturing cost and complexity of these electromagnets. Our goal has been to shrink the footprint of the single pixel to fit in a square $6 \mathrm{~mm} \mathrm{x}$ $6 \mathrm{~mm}$ to fit under a $6 \mathrm{~mm}$ diameter feedhorn aperture. However, the $20 \mathrm{~mm}$ x $5 \mathrm{~mm}$ footprint of a single electromagnet is almost 3 times the goal footprint for the entire KAPPa pixel, see Figure 1. Despite the many disadvantages, the 
electromagnet's field can be tuned at any time during the operation of the receiver. As discussed in Section 4.1, by tuning the receiver's magnetic field we can make a map of receiver performance as a function of magnetic field strength.

The magnetic field of the electromagnet is generated by a wrapped solenoid of superconducting wire. A DC current on superconducting wire does not produce a thermal load on the KAPPa cryostat's $4.2 \mathrm{~K}$ stage. However, the low thermal conductivity cryowires proceeding the superconducting magnet wire produce a larger heat load than all other receiver components combined, including the first stage low noise amplifier (LNA). Smaller currents can be used if the solenoid has additional wraps, but this increases the cost, complexity, and size of the electromagnet. Due to their physically smaller SIS junctions, the KAPPa SIS devices require a higher magnetic field strength than their SuperCam counterparts. The SuperCam electromagnets were machined using high magnetic permeability metals. After machining the electromagnet components are wrapped with superconducting wire. This process is expensive and time consuming and the result is an electromagnet that is several times larger than all the other pixel components combined. To make an array of $\sim 1000$ SIS pixels, with densely packed pixels, we must shrink the size of the magnet system.

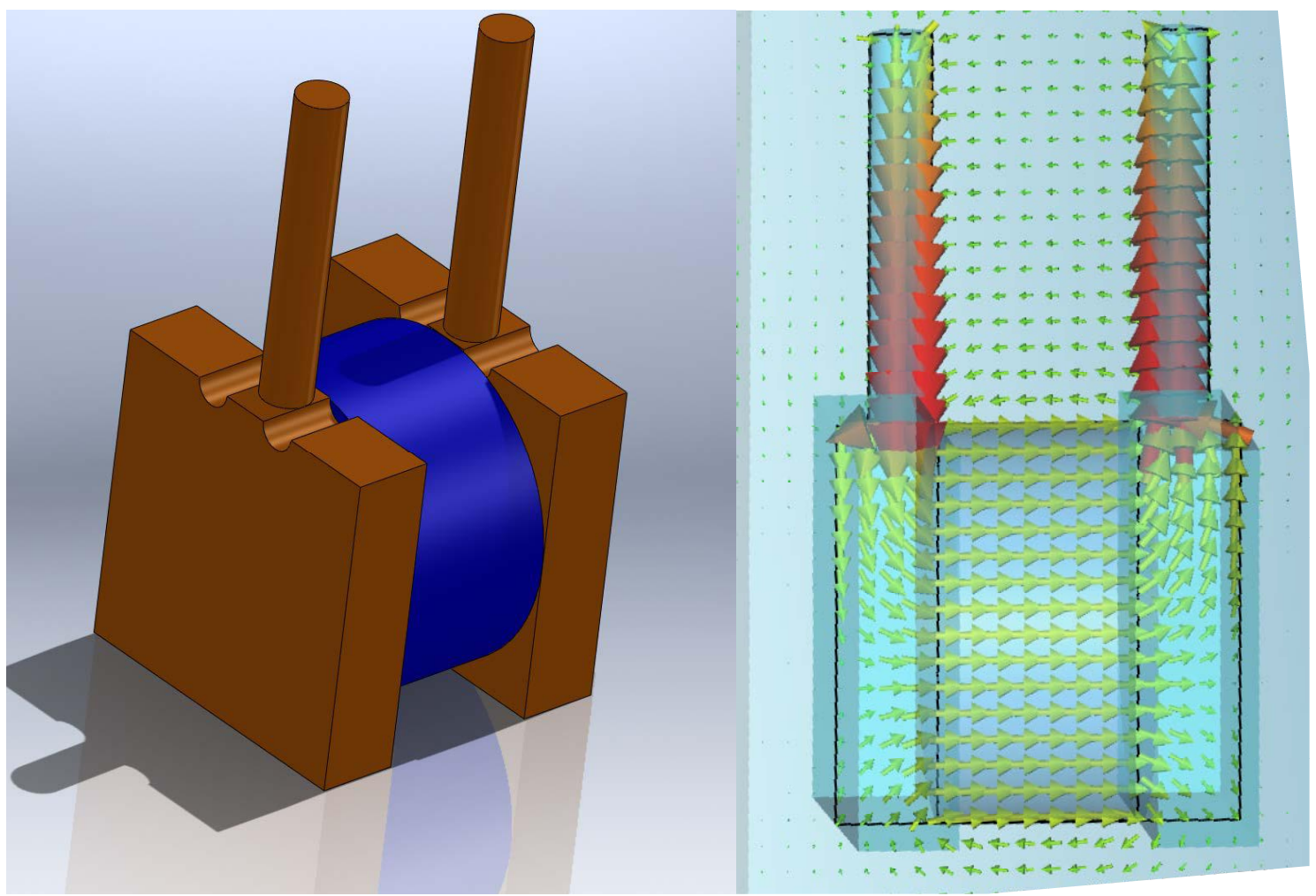

Figure 2. The permanent magnet system. The left side shows a solid model of the permanent magnet system. The permanent magnet is cylindrical commercially available magnet shown in blue. The magnetic field concentrators, (ping-pong paddles) are shown in orange. The narrow 'handles' of the field concentrators increase the magnetic field over the SIS junction. The right side shows the magnetic field vectors for the permanent magnet system simulated in CST.

\subsection{Permanent Magnet Design}

Using the feedhorn aperture as the maximum footprint size of a pixel, it was required that all of the pixel components fit into a $6 \mathrm{~mm} \times 6 \mathrm{~mm}$ cell. Our original design called for the electromagnets to be placed in the same plane as the SIS device and the LNA. Even with strong rare earth neodymium magnets, the field strength and orientation required for the SIS device called for magnets that were too large to fit within the $6 \mathrm{~mm}$ by $6 \mathrm{~mm}$ cell. This issue was mitigated by using a ' $U$ ' shaped permanent magnet system (see Figure 2) that is installed from the rear of the pixel, the side of the detector block that was opposite of the feedhorn, as shown in Figure 1. With this improvement, the plane of the SIS device only needs to have two small holes that the legs of the ' $U$ ' shaped permanent magnet system pass through. We were able to make the legs of the permanent magnet system thin, allowing the legs to be placed closer to SIS device decreasing the required strength of the permanent magnet. This design has three advantages: 1) The desired decrease in pixel footprint 
size; 2) There was more room for different permanent magnet geometries, allowing us to select from a wider variety of magnet sizes and strengths to provide the required field strength; and 3) The effects of a pixel's magnet are minimized on neighboring pixels since alternating the magnetic field direction for neighboring pixels can further minimize the effects of neighboring magnets has on a given pixel.

Magnets are difficult to machine because they are brittle and the magnetized chips that form during the machining process will be attracted to any ferrous metal used in the machining process. Therefore, it is preferable for both cost and simplicity of design to utilize unmodified commercially available magnets that are a standard. To reshape the magnetic field for the KAPPa SIS device, we machine steel concentrators to make the legs of the ' $U$ ' shaped permanent magnet system, see Figure 2. The 1010 steel has a high magnetic permeability and can have its magnetic properties simulated in finite element codes. The final permanent magnet system design utilized a cylindrical permanent magnet and two steel field concentrators shaped like ping-pong paddles. The large, flat 'paddles' of the concentrators sandwiches the permanent magnet, while the two thin handles extend into the plane of the SIS device channeling the magnetic field to reconnect across the SIS device (see Panel C in Figure 1).

\subsection{Permanent Magnet Simulation}

Microassembly of the components in a heterodyne pixel is a tedious process, thus the alignment of $\sim 1000$ permanent magnets in a future heterodyne arrays should be as simple as possible. With this in mind, the permanent magnet system should produce a magnetic field that change by less than $10 \%$ for any possible misalignment of the SIS device or the permanent magnet system. We used Computer Simulation Technology (CST) to evaluate various geometries of permanent magnet system with the goal of designing a magnetic field is as flat as possible in the region of the SIS device.

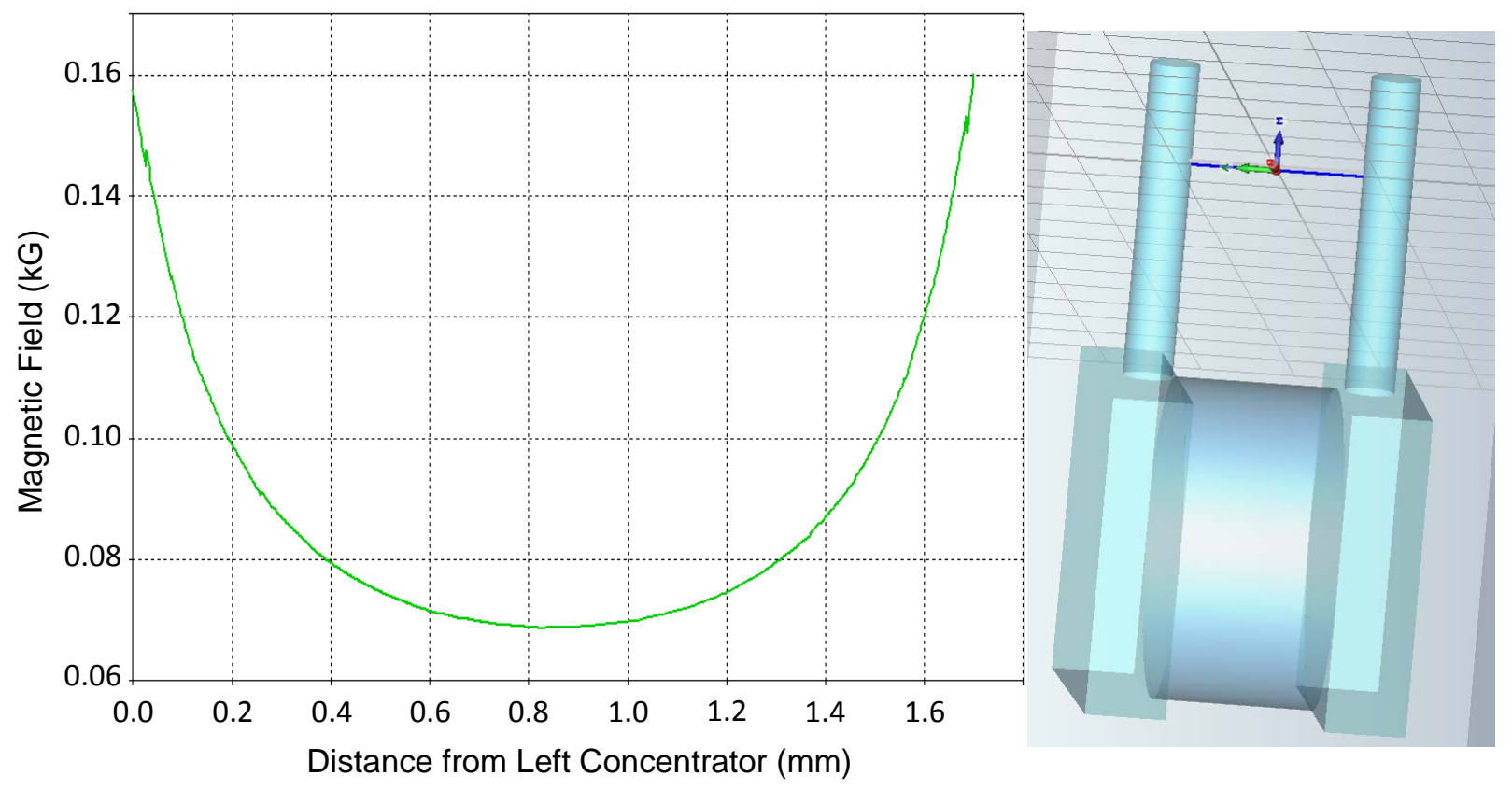

Figure 3. The magnetic field for the permanent magnet system across the SIS device. The right image shows the geometry of the permanent magnet system. The magnetic field strength is measured across the path of the blue line that connects the left and right field concentrators. The center of this path is the location of the SIS device at $0.85 \mathrm{~mm}$ from each concentrator. The plot on the left side shows the magnetic field strength along the path of the blue line in the right image.

During the CST evaluations, our goal was to having a uniform magnetic field in a region around the SIS device that was 1.5 times that of the maximum mechanical tolerances for both the alignment of the SIS device as well as the permanent magnet system. The maximum mechanical tolerances for the microassembly of the SIS device and permanent magnet system were less than $0.05 \mathrm{~mm}$. Figure 3 shows the field strength between two concentrators on a path the goes through the SIS device. We were able to achieve desired field flatness for a length scale greater than that of the mechanical tolerances by extending the yokes above the plane of the SIS device and LNA by $1.0 \mathrm{~mm}$. The field flatness could have 
been improved by making the area of the field concentrators leg larger for the side facing the SIS device, but the conclusion of our trade study indicated that it was more desirable to have a thin yoke leg that could be brought as close to SIS junction as possible.

Our simulation results informed us of the gradient and the approximate field strength that we could expect based on the data sheets from our commercially available magnets. Simulation helped to choose an effective geometry that would be tolerant of misalignment. After finding an effective geometry using CST evaluations we would then determine what could be mechanically designed. Mechanical design and magnetic field design required many iterations before all design criteria were achieved. Small pieces and tight tolerances are good for the magnetic field design but can be expensive to machine. A future design might benefit from much larger pass-through for concentrators in the detector block which lead to smaller, shallower features in the horn block. This would make the detector block features easier to machine while still maintaining the tight mechanical tolerances by capturing the tops of the magnet concentrators in the horn block. Simulation was done under the expectation that real magnets would exhibit larger than advertised field strengths that varied from magnet to magnet.

\section{PROCEDURE FOR MAGNETIC FIELD TESTING PRIOR TO INSTALLATION}

\subsection{Measurements of Magnetic Field Strength}

To measure the magnetic field strength of the permanent and electromagnets, we used a commercially available gaussmeter. This was the model 5180 Gauss/Tesla Meter by F. W. Bell. The entire device consisted of a handheld meter with digital output display, and a selection of detachable probes. The field strength was measured using the transverse Hall probe, which allows us to take measurements of the magnetic flux density between magnetic poles. Correct orientation of the encased Hall Effect sensor within the transverse probe was necessary to achieve flux measurements near and in-between the field concentrators of the permanent magnets. The probe itself was a thin plastic stem only 1.14 $+/-0.1 \mathrm{~mm}$ thick housing the small sensor which is located $0.85+/-0.1 \mathrm{~mm}$ from the tip of the probe. The diameter of the active sensor area for the transverse probe is $0.38 \mathrm{~mm}$ as given by the manufacturer. This active area of flux being measured is significantly larger than the true area of flux passing over the $1 \mu \mathrm{m} \times 1 \mu \mathrm{m}$ SIS junction.

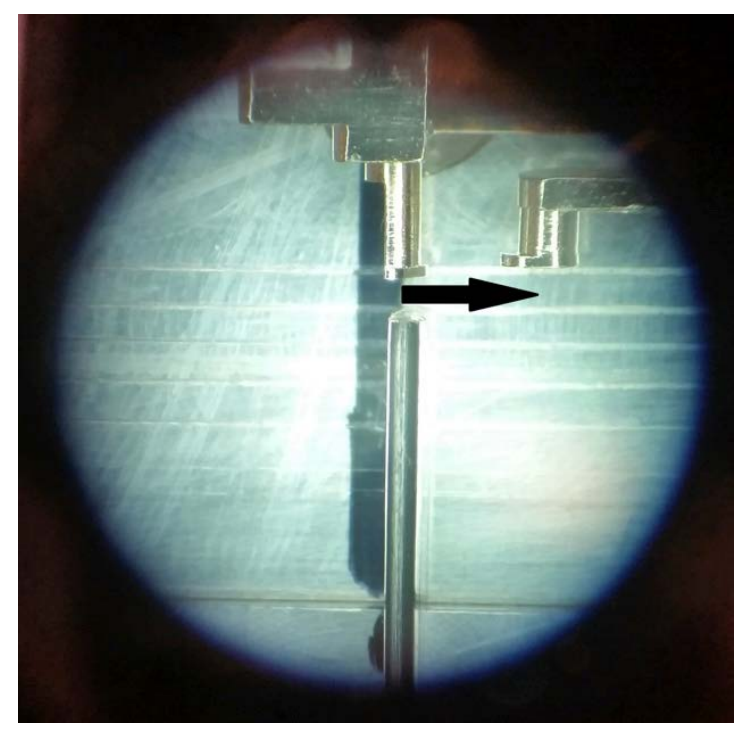

Figure 4. Microscope image of the electromagnet and gaussmeter. A sweep of the magnetic field is performed while the electromagnet is stepped horizontally on a precision platform compared to a fixed transverse sensor probe of the gaussmeter The direction of probe sweep is indicated by a black arrow, and the engraved lines on the moving platform mark the distance from concentrators at $1 \mathrm{~mm}$ intervals.

The measurements of magnetic field strength required keeping the Hall element within the gaussmeter normal to the incoming flux lines. It was important to move the probe as little as possible during measurement to minimize contamination from ambient magnetic fields. An aluminum precision motion stage was required to keep the stage form 
being magnetized. It proved extremely challenging to measuring the magnetic fields of the small magnets used in the KAPPa receiver, and the region where the SIS junction is located is even smaller. The regions that we were attempting to measure were much smaller than the characteristic size of the probe used for measurement. The only way to get around this issue of scales was to make many measurements at very small steps and then repeat this process a statistically significant number of times.

Using a microscope and the engraved scale on the platform, the probe and concentrators were brought to a specific distance, which was often less than or equal to $1 \mathrm{~mm}$ apart. When the field concentrators are attached to our cylindrical neodymium magnets, there is only $1.7+/-0.1 \mathrm{~mm}$ of separation between the concentrators. This presents a challenge since the sensor probe thickness, compared to the spacing of the concentrators, leaves little room for movement. Very few data points can be gathered in this space because the probe can collide with the magnet potentially causing damage the sensor probe, or simply changing the orientation of the probe with the magnet. To give an estimate of values for the magnetic field strength between concentrators, a single data point was measured for several permanent magnets by centering the probe at the location between the poles where the field would be redirected over the SIS junction in the final pixel. However, this would not be enough to give us confidence in the permanent magnet. A second set of measurements was taken at the tip of the iron concentrators. Here the probe would scan laterally from one concentrator to the next to determine the relative field strength and uniformity, which should exhibit similar behavior as the field lines between concentrators as it does a known distance away. Once the full distance was traveled, the magnet was sent back to starting position and the sweep was repeated several times. This was done for consistency and to verify reproducibility. Four identical permanent magnets were measured in this fashion. Figure 4 shows a microscope image of the probe sweeping procedure for the electromagnet.

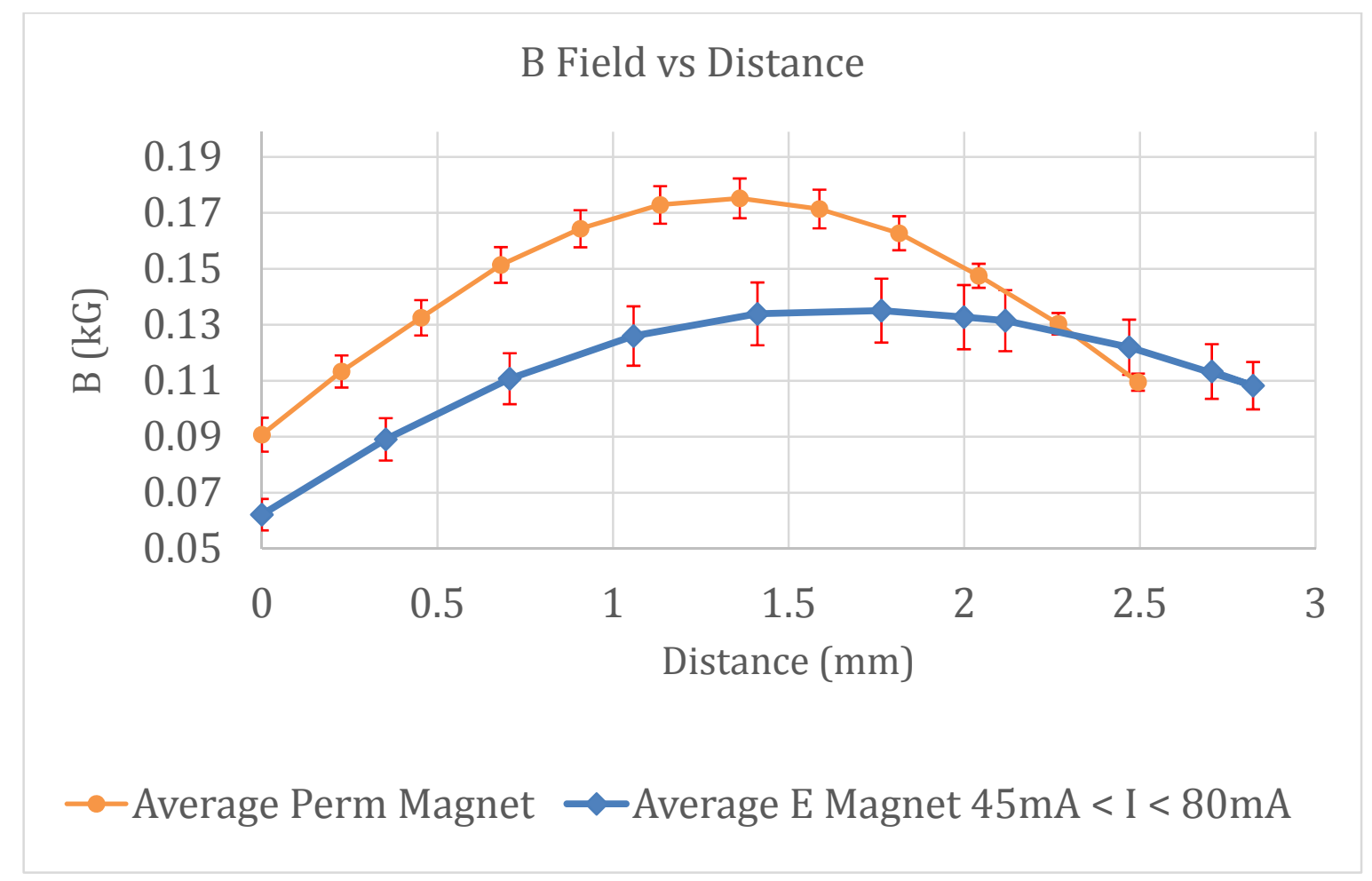

Figure 5. The average flux density of a set of 3 identical permanent magnets (data points with filled circles) over a single sweep between concentrators. The average flux density of an electromagnet with current between $45 \mathrm{~mA}$ and $80 \mathrm{~mA}$ is shown with filled squares. The distance in $\mathrm{mm}$ is measured from the center of one concentrator to the next, while the separation of the sensor to the concentrators is $0.85+/-0.1 \mathrm{~mm}$.

The electromagnets were tested in a similar fashion. The base of the electromagnet was bonded to the platform and the leads were connected to a variable power supply. The power supply let us measure the magnets for a range of currents starting from $45 \mathrm{~mA}$ and leading up to $80 \mathrm{~mA}$. The typical operating current of the electromagnet, when installed in the KAPPa receiver, was $65 \mathrm{~mA}$. The chosen spread in current allowed us to observe the electromagnet at range of field 
strengths that could be expected in the receiver. The concentrators on the electromagnet were spaced further apart than the ones in the permanent magnet. The electromagnet had one concentrator that was much shorter than the other (seen in Figure 4) creating an asymmetric magnetic field strength (seen in Figure 5). Because of the asymmetry, the field strength at the center of the concentrators varied more than the permanent magnet systems adding to the difficulty of comparison. The asymmetry of the electromagnet affects the flux density at the ends of its concentrators, which makes it difficult to compare the uniformity of the permanent magnet systems. Figure 5 shows that the permanent magnet system exhibits a symmetric behavior with a uniform central field, not unlike simulation. Because the permanent magnet demonstrated generally higher flux densities then required, we attempted to lower the magnetic field of the permanent magnets as discussed in Section 3.2.

\subsection{Determining the Required Magnetic Field Strength}

Permanent magnets can have their magnetic field reduced by heating them to their Curie temperature $\left(\mathrm{T}_{\mathrm{C}}\right)$ for small amounts of time. In this way we are able to purchase magnets that have a stronger field then required and heat them until they have exactly the desired field. The technique was also used to demagnetize any of the iron field concentrators that may have been in too strong of an applied field. The $\mathrm{T}_{\mathrm{C}}$ for our cylindrical neodymium magnets was $310^{\circ} \mathrm{C}$. An ordinary hotplate was used for the procedure of weakening the magnets. Once the hotplate was warmed to $\mathrm{T}_{\mathrm{C}}$, a single magnet was placed at the center. With constant heating for only 5 seconds at $T_{C}$, the flux density of the heated magnet was reduced to $60 \%$ of its original value. Following the first successful KAPPa test using a permanent magnet a second permanent magnet was heated in this same way and brought to $63 \%$ of the strength of first magnet.

Prior to the measurement of the electromagnet field strength, several sets of permanent magnet systems were constructed. Each system has a different magnetic field strength corresponding to a different round of heating at the magnet's $T_{C}$. We then compared that field strength of the sets of permanent magnet systems to the measured electromagnet field under typical operating currents. Based on our observations from sweeping the electromagnet (discussed in Section 4.1) we know that there is a zone of infinite system temperature that is seen for weak magnet fields. Therefore, it was prudent to select a permanent magnet system with a stronger field than that what had been determined to be ideal at the lab bench.

\section{MAGNET PREFORMANCE IN THE KAPPA RECIEVER}

\subsection{Electromagnet Results}

In this section, magnetic field strength $(|\mathrm{B}|)$ is discussed in terms of current through the solenoid (a component of the electromagnet system), in lieu of absolute calibrated field strength. While the absolute field through the SIS junction is calculable, it relies on measurements spatial changes in the magnetic field that are smaller than the probe of our gaussmeter. In addition, we are only able to make $300 \mathrm{~K}$ measurements of the magnetic field on a lab bench, which may not fully correspond to in situ measurements at $4.2 \mathrm{~K}$ where the magnet permeability of materials increases. Thus we present solenoid current $\left(\mathrm{I}_{\mathrm{S}}\right)$ as proxy for $|\mathrm{B}|$.

Figure 6 shows the best receiver system temperatures $\left(\mathrm{T}_{\text {sys }}\right)$ as a function of the electromagnet solenoid current $\left(\mathrm{I}_{\mathrm{S}}\right)$. As seen in Figure 6, there are several minima for $T_{\text {sys }}$ as a function of $I_{S}$. As $I_{S}$ increases, the range of $I_{S}$ per $T_{\text {sys }}$ minima increases and $T_{\text {sys }}$ between the minima decreases. In practice, the first minima for $T_{\text {sys }}$ is not useful for setting the receiver. This is due to the hysteretic effects in $\mathrm{I}_{\mathrm{S}}$ when setting magnetic fields that are larger than range of currents that fall in the $1^{\text {st }}$ minima. Setting the electromagnet at the first minima required slowly stepping the electromagnet current and halting the stepping when optimal $\mathrm{T}_{\text {sys }}$ had been achieved and requires two sweeps if the optimal $\mathrm{T}_{\text {sys }}$ is not known. The other minima were more tolerant of variation in the magnetic field strength. For ease of use, we commonly set the electromagnet to the fourth minima in Figure 6, corresponding to $I_{S}$ greater than $50 \mathrm{~mA}$. Because $T_{\text {sys }}$ between minima decreases as $I_{S}$ increases, the receiver is more likely to have measurable response for non-optimal fields near the fourth minima. This was an important consideration when tuning the permanent magnet field strength.

\subsection{Permanent Magnet Results}

During the first permanent magnet test on the KAPPa receiver, we could not predict how the magnet field strength might change as the permanent magnet system was cooled to $4.2 \mathrm{~K}$. To maximize the likelihood of a positive result, we attempted to calibrate our permanent magnet to be in the fourth minima for $\mathrm{T}_{\mathrm{sys}}$ (the 50-62 $\mathrm{mA}$ range in Figure 6). For 
the fiducial parameters of LO frequency of 670 and $675 \mathrm{GHz}$ at a $15 \mathrm{MHz}$ IF bandwidth centered at $2 \mathrm{GHz}$, the first permanent magnet yielded a system temperatures of near $200 \mathrm{~K}$. Figure 6 shows the best system temperatures achieved with the electromagnet in the 170-180 K range. We concluded that we had likely over-shot the magnetic field strength for the $1^{\text {st }}$ permanent magnet system. The $2^{\text {nd }}$ permanent magnet system was designed and measured to have a $63 \%$ reduction in field strength compared to the $1^{\text {st }}$ permanent magnet system.

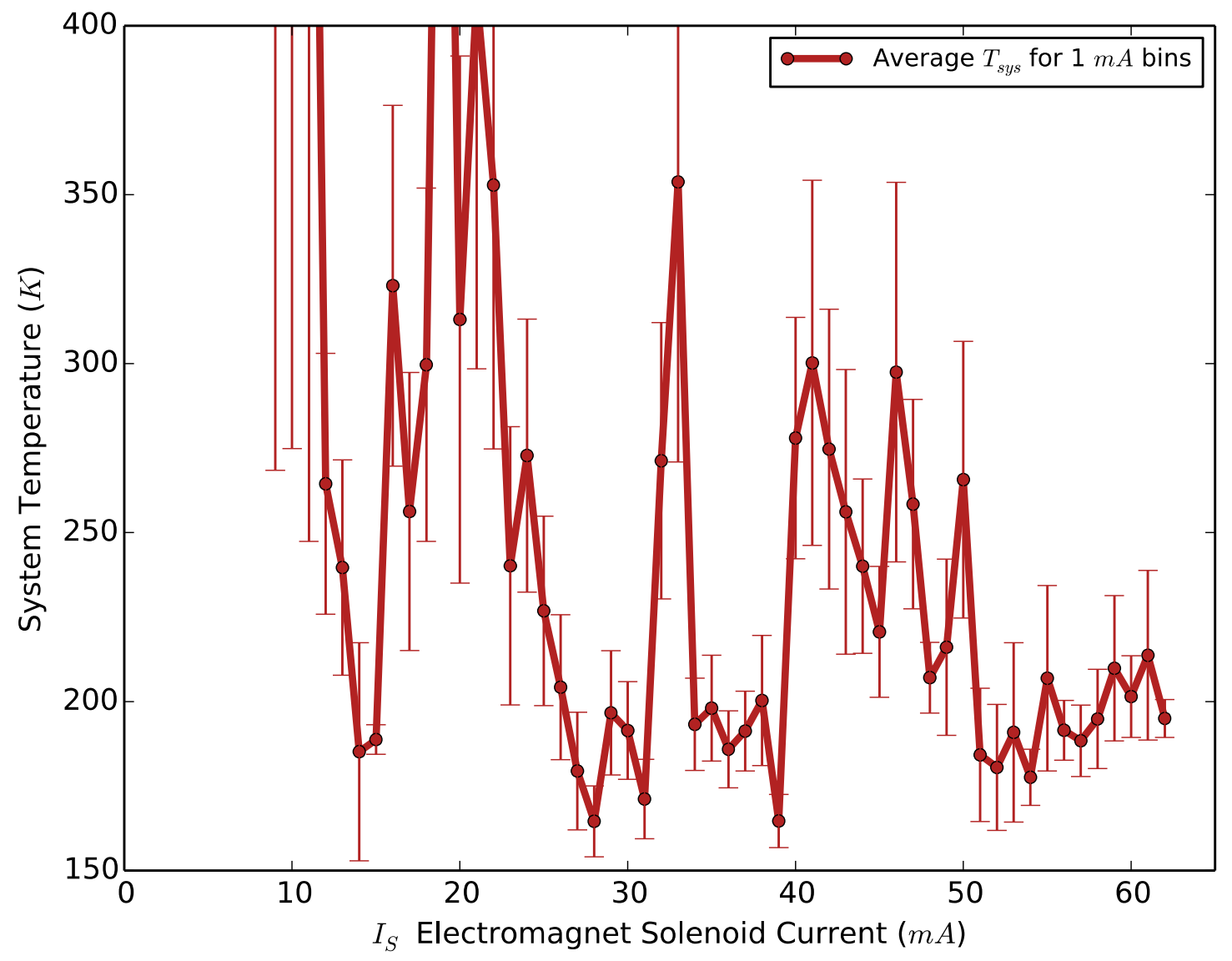

Figure 6. System temperature as a function of electromagnet current. The error bars show the spread of the 5 points per 1 $\mathrm{mA}$ bins. These points were taken using the center LO frequency of $670 \mathrm{GHz}$ and at a $15 \mathrm{MHz}$ IF bandwidth centered at 2 $\mathrm{GHz}$. We can see several minima in system temperature, at $14 \mathrm{~mA}, 28 \mathrm{~mA}, 37 \mathrm{~mA}$, and $54 \mathrm{~mA}$. As the electromagnet current increases the minima can be found over larger ranges of magnet current. Using this data, larger magnet field strengths for the permanent magnets are more likely to display low system temperature even in case non-optimal field strength

In Figure 7, we compare the electromagnet results to those of the two permanent magnet systems for system temperature and a function of LO frequency. The constituent data for the electromagnet and $1^{\text {st }}$ permanent magnet were created using a searching algorithm that allowed the LO frequency to be any rational number, while the $2^{\text {nd }}$ permanent magnet data is a simple sweep of integer values of LO frequency [5]. Since the LO does not have uniform amplitude modulated noise performance as a function of frequency, there were times when the searching algorithm could find lower system temperatures for $\mathrm{LO}$ frequencies near, but not on, the integer value of frequency. In this way, better system temperatures for the less optimized field of the $1^{\text {st }}$ permanent magnet could be achieved over the more optimized field of the $2^{\text {nd }}$ magnet.

The $2^{\text {nd }}$ permanent magnet system greatly improved the KAPPa receiver's ease of use compared to the previous electromagnet and permanent magnet. It became challenging to find settings that were non-optimal. During this final configuration of the KAPPa receiver, there were multi-hour periods of time where the receiver operation at system temperatures that were lower than what had ever been achieved with the electromagnet (Figure 7, diamond markers from 
$675-690 \mathrm{GHz}$ ). During the KAPPa experiment, measurements of the current across the SIS device were used to tune the LO power. For the $2^{\text {nd }}$ permanent magnet, we found that the optimal current across the SIS device was lower then it had been for electromagnet and $1^{\text {st }}$ permanent magnet. We hypothesis that this is the result of better suppression of Josephson noise by the field of the $2^{\text {nd }}$ permanent magnet, which also highlighted the need to reapply our optimization algorithm after any changes in hardware [5].

Overall the permanent magnet performance was within $50 \mathrm{~K}$ of the electromagnet performance (Figure 7) and improvements in the permanent magnet performance are still possible. The ease of operation for the KAPPa system was greatly increased with the installation of permanent magnets. Because of hysteretic effects, the electromagnet was the most difficult input parameter of the KAPPa system to tune. With the dominant heat load from the electromagnet current gone, the KAPPa cryostat doubled helium hold time. The installation of the permanent magnet was a major boon for the receiver operations team. The operation of the receiver was simplified without the need to tune the electromagnet. A full characterization of the receiver dropped from weeks of testing to only 3 days, and the hold time of the receiver's cryostat was doubled without the added heat load from the electromagnet.

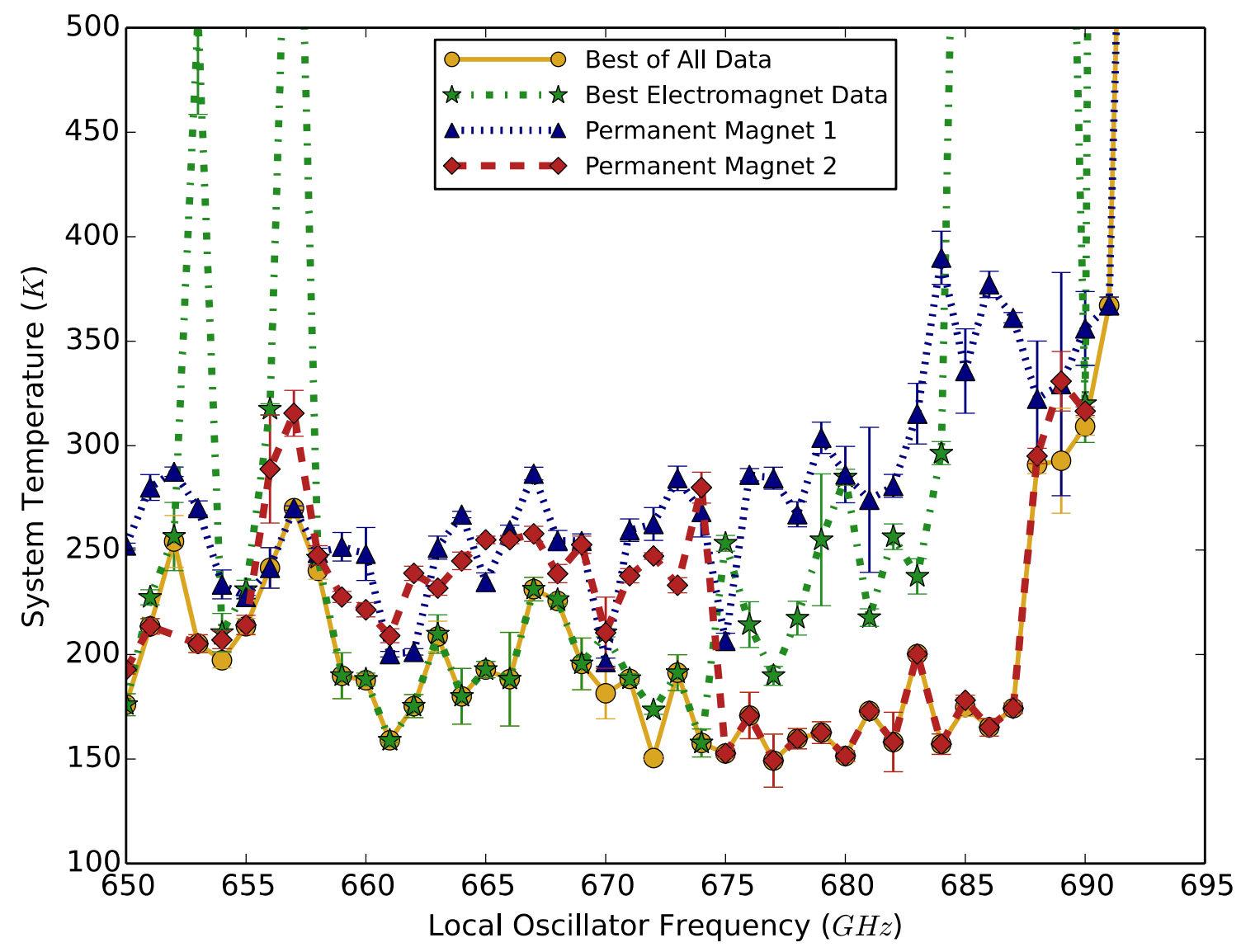

Figure 7. Best system temperatures as a function of LO frequency. The solid line with circle markers is the best of all data for the entire KAPPa experiments. The dot-dashed line with star markers is the best of all electromagnet data, the electromagnet allows for precise tuning of the magnetic field. Permanent magnet 1 and Permanent magnet 2 are the triangle dotted and diamond dashed lines respectively. Each data point is calculated from the mean of the 5 best data points in a 1 $\mathrm{GHz}$ bin, the error bars represent the standard deviation. All points are filtered to be within the expected SIS bias voltage and current ranges. Additional filtering removed single measurements with system temperature standard deviations greater than $10 \%$. Only permanent magnet 2 data was taken at integer values of LO frequency, other data has components that were created by a search algorithm that was allowed to have any rational LO frequency. Electromagnet data was taken using a thinner beam splitter. The thinner beam splitter accounts for high system temperatures at the extremes of the LO tuning range due to insufficient $\mathrm{LO}$ power. 


\section{REFERENCES}

[1] Leridon, B., Josephson Noise in SIS Receivers, Proc. ISSTT 5, p558, (1994)

[2] Groppi, C., et al., Testing and Integration of Supercam, a 64-Pixel Array Receive for the $350 \mathrm{GHz}$ Atmospheric Window, Proc. SPIE 7741, 774110X, (2010).

[3] Groppi, C. E. et al., The Kilopixel Array Pathfinder Project (KAPPa), a 16 pixel integrated heterodyne focal plane array, Proc. SPIE 8452, 84520Y, (2012)

[4] Wheeler, C. H., et al., The Kilopixel array pathfinder project (KAPPa), a 16-pixel integrated heterodyne focal plane array: characterization of the single pixel prototype, Proc. SPIE 9153, 91530K, (2014)

[5] Wheeler, C. H. \& Toland, T. G., Sufficient Receiver Tuning Using Differential Evolution Strategies, Proc. SPIE (2016), submitted 